\title{
Pneumonectomy in a Patient with Swyer-James-MacLeod Syndrome
}

\author{
Mariana Vigiola Cruz ${ }^{1}$, Gregory D. Trachiotis ${ }^{1,2^{*}}$ \\ ${ }^{1}$ The Division of Cardiothoracic Surgery, Veterans Affairs Medical Center, Portland, USA \\ ${ }^{2}$ The George Washington University, Washington DC, USA \\ Email: "Gregory.Trachiotis@va.gov, ${ }^{*}$ gtrachiotis@mfa.gwu.edu
}

Received 4 January 2015; accepted 8 February 2015; published 11 February 2015

Copyright (C) 2015 by authors and Scientific Research Publishing Inc.

This work is licensed under the Creative Commons Attribution International License (CC BY).

http://creativecommons.org/licenses/by/4.0/

C. (i) Open Access

\begin{abstract}
Swyer-James-MacLeod syndrome is a rare disease that results in parenchymal damage believed to be caused by recurrent lung infections in childhood. We report a case of a previously healthy young woman that presented with insidious progression of exertional dyspnea with restrictive lung physiology. Axial imaging demonstrated a hyperinflated emphysematous right lung with lower lobe fibrosis, nodules and air-fluid filled cysts, with a small right pulmonary artery and contralateral shifting of the mediastinum. She underwent right pneumonectomy ultimately resulting in improvement of her symptoms, with surgical pathology indicating extensive emphysema, bronchiectasis, fibrosis with osseous metaplasia, and placental transmogrification of alveolar septa, being consistent with a diagnosis of SJMS.
\end{abstract}

\section{Keywords}

\section{Swyer-James, Hyperlucent Lung, Pneumonectomy}

\section{Case Report}

A 26-year-old female presented with new-onset exertional dyspnea and decreased exercise endurance over the preceding year. She was otherwise asymptomatic and denied further constitutional, cardiac, pulmonary, or systemic symptoms. On physical examination she was a well-developed and nourished female, with normal vital signs and pulse-oximetry. Marked decrease in airflow over the entire right hemithorax and inspiratory crackles in the right base were noted. The remainder of her physical examination was within normal limits.

In her history, she denied recurrent episodes of pneumonia or bronchitis, describing only a prolonged hospitalization during infancy for a critical case of meningitis, without any known pulmonary complications. She was a

${ }^{*}$ Corresponding author. 
lifelong non-smoker and was an active athlete in adolescence. At age 18 during induction of anesthesia for breast reduction surgery she became acutely hypoxic leading to cancellation of the procedure. A subsequent chest radiograph showed unilateral hyperlucent lung, with pulmonary function tests showing mild restriction. She was given a clinical and radiographic diagnosis of SJS and was followed non-operatively without interval complications for several years.

On presentation to our institution, a chest radiograph indicated a hyper-expanded right lung with right-to-left mediastinal shift, right basilar fibrosis with an air-fluid-filled cyst in the right lower lung field, and an inferiorly displaced right hemidiaphragm. A CT of the chest demonstrated a severe hyperinflated, emphysematous right upper and middle lung, partial preservation of the right lower lung with diffuse fibrosis, nodules and cystic air-fluid-filled cavities, and small but patent right main stem bronchus and pulmonary artery (Figure 1). The left lung was small with normal appearing parenchyma. Pulmonary function tests showed restriction (TLC 66\% predicted) without obstruction (FVC 59\%, FEV1 52\% predicted), and decreased diffusion (DLCO 57\% predicted). An echocradiogram depicted normal chamber dimensions with preserved systolic and diastolic function without evidence of pulmonary hypertension. A scintigraphic ventilation perfusion scan determined the right lung to be responsible for $15.9 \%$ of the ventilation and $11.5 \%$ of the perfusion, corresponding to a matched defect.

A decision was made to proceed with pneumonectomy considering her functional limitation, risk for progressive degeneration of the lung and cardiopulmonary status, the possibility of chronic infections, abscess formation and hemorrhage, and the potential negative impact in future pregnancies. The surgical approach was through a modified muscle sparing right thoracotomy with a posterolateral incision. The gross lung specimen was 2 to 3 times normal, as shown in Figure 2. The pathology demonstrated extensive panacinar emphysema, bronchiectasis, fibrous scarring with osseous metaplasia, and placental transmogrification of alveolar septa, consistent with a diagnosis of Swyer-James-Macleod syndrome. There were no operative complications and the postoperative course was unremarkable.

Following recovery the patient reported symptomatic alleviation with pulmonary function tests reflecting improved restriction, diffusion, and flow (TLC 74\%, DLCO 61\%, FVC 69\%, FEV1 75\% predicted). Two years after operation, the patient required a mediastinal neutralization procedure for post-pneumonectomy syndrome, and has remained symptom free thereafter.

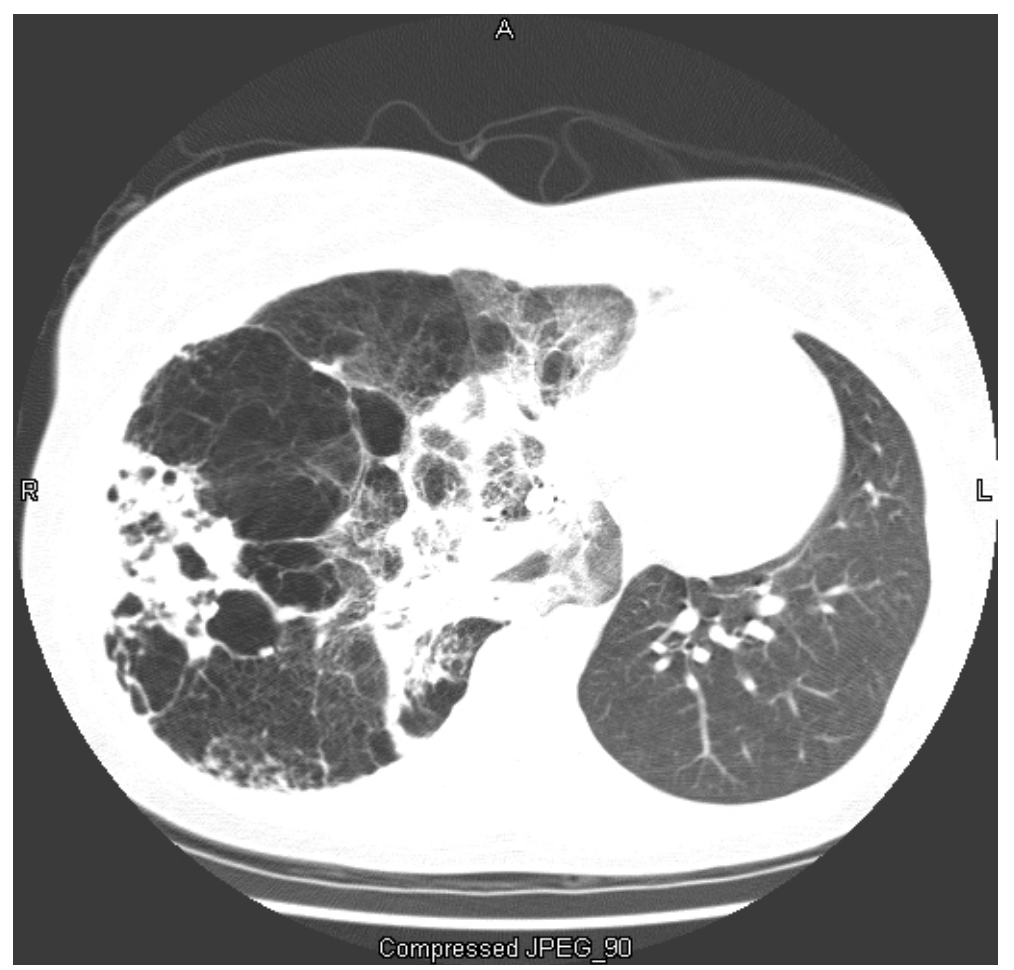

Figure 1. Chest computed tomography demonstrating hyper-inflated, cystic and fibrotic right lung changes. 


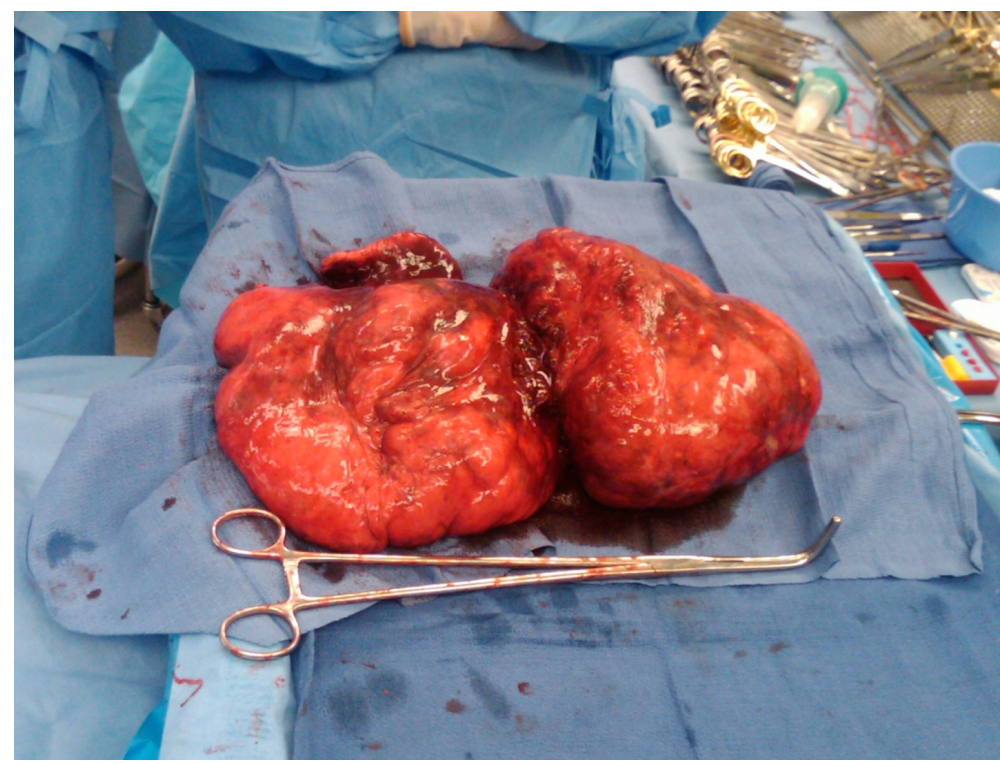

Figure 2. Gross pathology of right pneumonectomy specimen with lung volume size 2 - 3 times normal.

\section{Discussion}

Swyer-James-MacLeod syndrome (SJMS) or unilateral hyperlucent syndrome is an uncommon disease believed to occur as a result of infectious bronchiolitis obliterans in infancy or early childhood. Initially described in 1953Swyer and James in pediatric patients [1] and a year later in several adults by McLeod, it has been reported to have a prevalence of $0.01 \%$ [2]. Respiratory viruses, mycoplasma pneumoniae, and pertussis have been commonly implicated as the insulting pathogens. Primary infection leads to destruction of the bronchial epithelium and adjacent parenchyma, causing impaired ciliary function and frequently leading to secondary or recurrent infections with further parenchymal damage. The resulting inflammation and fibrosis causes narrowing of the respiratory bronchiolar lumens reducing ventilation, and obliteration of the capillary beds, in turn also contributing to a decrease in perfusion [3]. Differential diagnoses include local emphysema, congenital hypoplastic or absent pulmonary artery, pneumatocele, bronchogenic cyst, sequelae of radiation therapy, bronchial carcinoma and benign intrabronchial neoplasm [3].

Patients generally report exertional dyspnea and/or chest pain, hemoptysis and chronic cough [1]-[3]. Alternatively, they may be asymptomatic with the radiographic abnormalities being identified incidentally in adulthood. These may include localized or diffuse emphysematous changes that are usually unilateral, with hyperexpansion of the affected lung with contralateral mediastinal shift, and bullous and cystic changes. Various degrees of interstitial fibrosis may be present. Air trapping may be seen on an expiratory PhaseComputer tomography, and bronchiectases are usually present, either saccular or cylindrical, with the latter more frequently associated with consolidation. The ipsilateral pulmonary artery may be hypoplastic. The unaffected lung may have normal parenchyma, be small in size and occasionally also be hyperexpanded and hyperlucent [3]-[5].

Pulmonary function testing frequently demonstrates obstruction with marked variation in severity between patients. Restriction can also occur and is usually a result of compression of the healthy lung by the hyper expanded diseased lung. The evolution of the airflow limitation is not known, but it is thought to remain stable over time in most patients. Ventilation perfusion scans have a characteristic pattern of a matched V/Q defect, poor vascular flow in the affected side, and air trapping on the washout phase.

There is no standard treatment for SJS, and clear indications for surgical management have not been well established. Management commonly concentrates in prevention, vaccine administration, early recognition and treatment of pulmonary infections, as well as alleviation of obstruction when present [3]. It is recommended that a surgical approach be contemplated if there are recurrent infectious complications or pneumothorax, worsening symptoms and deteriorating physiology. Literature reviews have identified fewer than 25 SJMS patients reported to have undergone lung resection for recurrent pulmonary symptoms [3]-[5]. Surgically treated patients 
have been noted to achieve clinical improvement by pneumonectomy, lobectomy or segmentectomy, bronchoscopic occlusion of the main bronchus without pneumonectomy, and VATS lung volume reduction surgery in case reports and small case series [3] [4]. In consideration of perioperative management, severe cases of SJMS may require anesthetic care cautiously tailored to significant obstructive pulmonary physiology.

Pathology examination of the lung usually reveals centriacinar and panacinar emphysema, bullous and cystic changes, fibrosis, interstitial pneumonia. Placental transmogrification is characterized by development of placental villi type structures in the lung parenchyma. It can be present in severe emphysema induced by smoking, congenital bullous emphysema, and fibrochondromatous hamartomas. I has been reported only once previously in Swyer-James syndrome [5].

\section{Summary}

We present a case of symptomatic Swyer-James-MacLeod syndrome successfully treated with pneumonectomy. Pointed clinical judgment is essential in diagnosis and management of this rare disease entity, for which the current literature still offers limited guidance. Prolonged follow-up is essential, as the residual pneumonectomy space may cause sequelae of mediastinal shift late recovery.

\section{References}

[1] Swyer, P.R. and James, G.C. (1953) A Case of Unilateral Pulmonary Emphysema. Thorax, 8, 133-136. http://dx.doi.org/10.1136/thx.8.2.133

[2] Macleod, W.M. (1954) Abnormal Transradiency of One Lung. Thorax, 9, 147-153. http://dx.doi.org/10.1136/thx.9.2.147

[3] da Silva, P.S., Lopes, R. and Neto, H.M. (2012) Swyer-James-MacLeod Syndrome in a Surgically Treated Child: A Case Report and Brief Literature Review. Journal of Pediatric Surgery, 47, e17-e22.

[4] Vishnevsky, A. and Nikoladze, G. (1990) New Approach to the Surgical Treatment of Swyer-James-MacLeod Syndrome. The Annals of Thoracic Surgery, 50, 130-134. http://dx.doi.org/10.1016/0003-4975(90)90098-Q

[5] Marchevsky, A., Guintu, R., et al. (2005) Swyer-James (MacLeod) Syndrome with Placental Transmogrification of the Lung: A Case Report and Review of the Literature. Archives of Pathology \& Laboratory Medicine, 129, 686-689. 
Scientific Research Publishing (SCIRP) is one of the largest Open Access journal publishers. It is currently publishing more than 200 open access, online, peer-reviewed journals covering a wide range of academic disciplines. SCIRP serves the worldwide academic communities and contributes to the progress and application of science with its publication.

Other selected journals from SCIRP are listed as below. Submit your manuscript to us via either submit@scirp.org or Online Submission Portal.
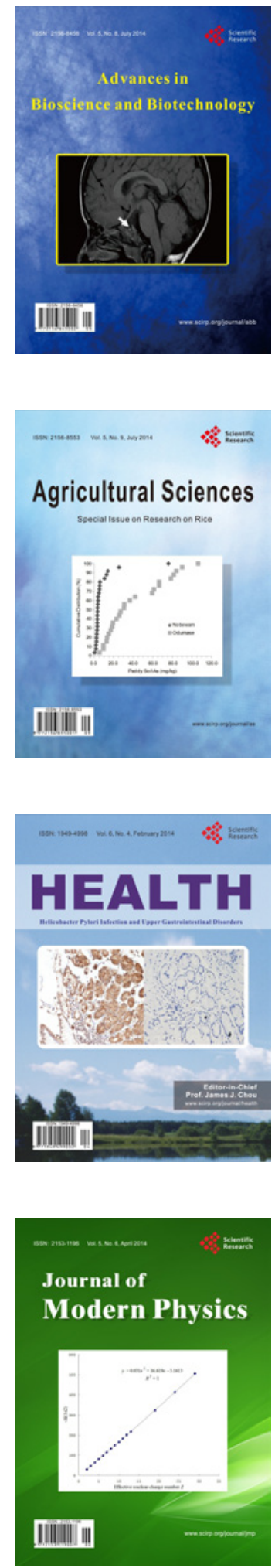
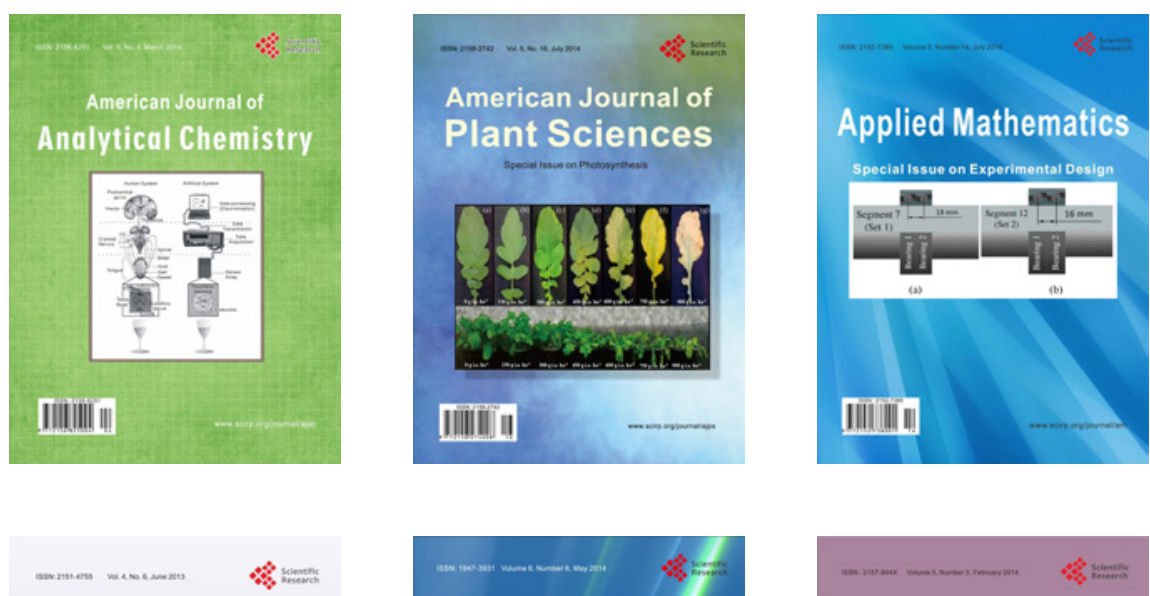

Creative Education
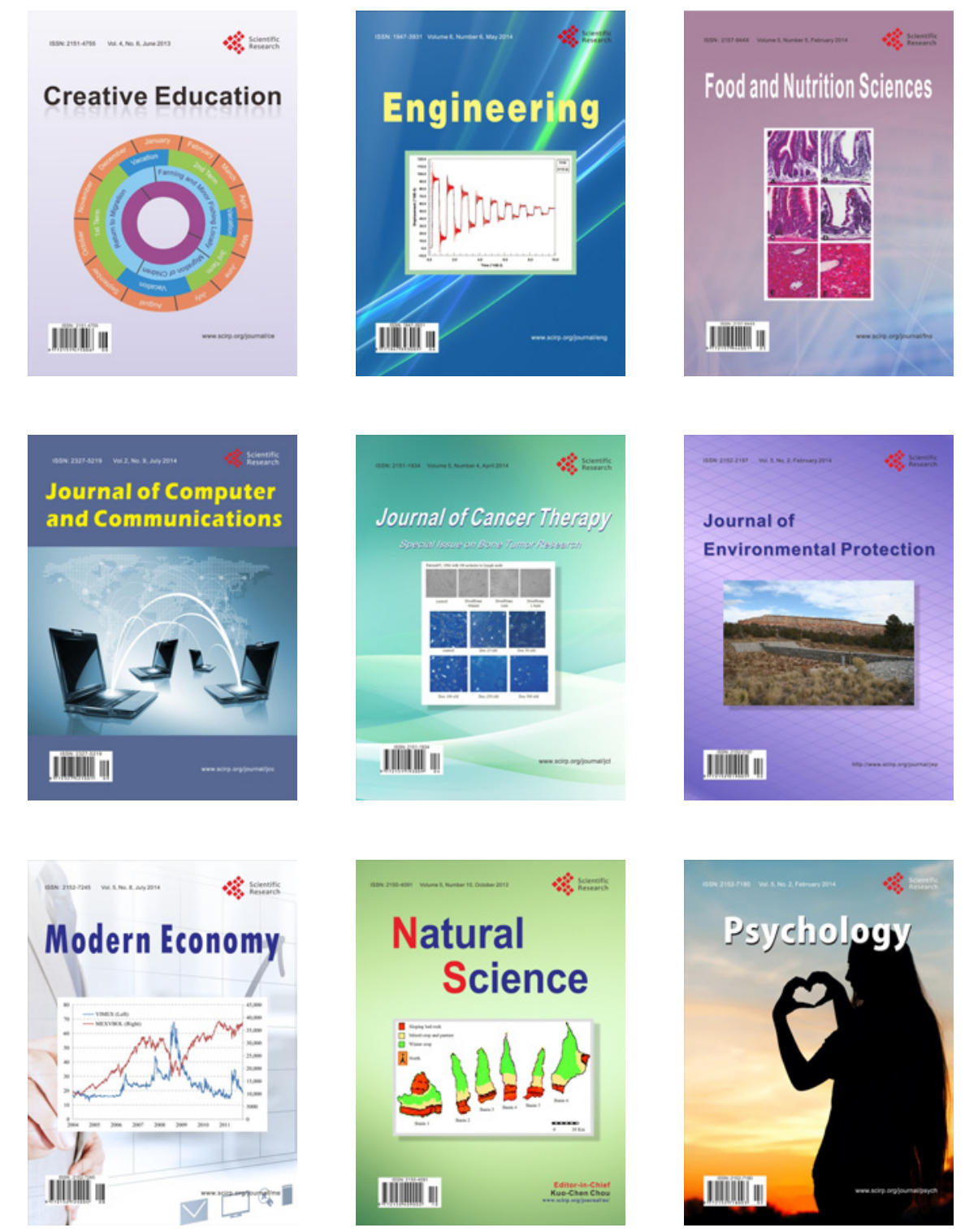